\title{
Inverse Nodal Problems for the Sturm-Liouville Operator with Some Nonlocal Integral Conditions
}

\author{
Xiaojuan Qin, Yunlan Gao*, Congmin Yang \\ College of Sciences, Inner Mongolia University of Technology, Hohhot, China \\ Email: *gaogaoyyyyy@sina.com
}

How to cite this paper: Qin, X.J., Gao, Y.L. and Yang, C.M. (2019) Inverse Nodal Problems for the Sturm-Liouville Operator with Some Nonlocal Integral Conditions. Journal of Applied Mathematics and Physics, 7, 111-122.

https://doi.org/10.4236/jamp.2019.71010

Received: November 29, 2018

Accepted: January 13, 2019

Published: January 16, 2019

Copyright $\odot 2019$ by author(s) and Scientific Research Publishing Inc. This work is licensed under the Creative Commons Attribution International License (CC BY 4.0).

http://creativecommons.org/licenses/by/4.0/

\begin{abstract}
In this paper, we study three inverse nodal problems for the Sturm-Liouville operator with different nonlocal integral conditions. We get the conclusion that the potential function can be determined by a dense nodal subset uniquely. And we present some constructive procedures to solve the inverse nodal problems.
\end{abstract}

\section{Keywords}

Sturm-Liouville Operator, Inverse Nodal Problems, Nonlocal Integral Conditions

\section{Introduction}

Sturm-Liouville operators have an important role in physics, mathematics and other engineering fields [1] [2] [3] [4] [5]. Nonlocal problems are parts of differential equation theory. And the inverse nodal problems are aimed at reconstructing operator by the given nodes (zeros) of characteristic functions. Lots of results were given by scholars.

Generally, Sturm-Liouville problems with nonlocal conditions have three kinds of form: potential with integral delay or function delay; nonlocal potential; nonlocal boundary conditions.

In 1988, Mclaughlin raised the inverse nodal problem for the first time. Applying a dense nodal subset, he got the uniqueness theorem of potential function [6]. In 1989, by the similar methods, some general boundary conditions and corresponding reconstruction of the potential function were provided by Hald and Mclaughlin [7]. In 2001, Xuefeng Yang discussed a new inverse nodal problem. By using Gesztesy and Simmon's inverse spectrum theorem, they proved 
that more than half of nodal subset could be used to uniquely determine the potential function [8]. In 2008, Hikmet Koyunbakan considered the Sturm-Liouville problem with some special boundary conditions. By using the Xuefeng Yang's method in [9], he studied the potential function's reconstruction and obtained a uniqueness theorem [10]. Chuanfu Yang studied a lot of inverse nodal problems. For example, in 2012, Yang studied the discontinuous inverse nodal problems. Applying the Riemann-Lebesgue lemma and Fatou's lemma, he solved the uniqueness, reconstruction and stability problems [11]. Through similar methods, in 2014, Yang studied inverse nodal problems with a constant delay. The uniqueness theorem was proved. And a constructive procedure was provided to solve the inverse nodal problem [12]. In 2017, Yang considered inverse problems on a graph with cycles. By the dense nodal points, the potential on a graph with multi-cycles can be constructed [13]. Besides, other scholars also studied the inverse nodal problems in recent years. In 2015, the discontinuous Sturm-Liouville operators' inverse nodal problems were discussed by Yuping Wang. By using the Green formula and Riemann-Lebesgue lemma, he established several uniqueness theorems [14]. In 2015, Juan P. Pinasco and Cristian Scarola studied an inverse problem for weighted Sturm-Liouville operators. By using weaker hypotheses for positive weights, the weight and the parameters were determined by a dense nodal set [15]. In 2018, Guangsheng Wei and Yongxia Guo provided the sharp conditions of the uniqueness for inverse nodal Sturm-Liouville problems. Applying a dense nodal subset, the potential and boundary parameters can be uniquely determined [16].

Based on [17], discussing the inverse nodal problems with some nonlocal integral conditions is the main content of this article. We conclude that a dense nodal subset can determine the potential function uniquely. And three constructive procedures for the solution of corresponding problems are proposed. Our main results of this paper are uniqueness theorems (see Theorem 3.4, 4.4, 5.4 below). It makes sense for the theoretical integrity of the inverse nodal problem with nonlocal integral conditions.

This paper's composition is as follows. In Part 2, we introduce preliminaries. In Part 3, 4, 5, we establish several uniqueness theorems for different boundary conditions.

Now let

$$
L y=-y^{\prime \prime}(x)+q(x) y(x)=\lambda y(x), \quad x \in[0, \pi]
$$

with nonlocal integral conditions

$$
\begin{aligned}
& y(0)=\int_{0}^{\pi} y(x) \mu_{0}(x) \mathrm{d} x \\
& y^{\prime}(0)=\int_{0}^{\pi} y(x) v_{0}(x) \mathrm{d} x \\
& y(\pi)=\int_{0}^{\pi} y(x) \mu_{\pi}(x) \mathrm{d} x \\
& y^{\prime}(\pi)=\int_{0}^{\pi} y(x) v_{\pi}(x) \mathrm{d} x
\end{aligned}
$$


where $q(x), \mu_{0}(x), \mu_{\pi}(x), v_{0}(x), v_{\pi}(x)$ are real-valued, continuously differential functions on $[0, \pi]$.

\section{Preliminaries}

Firstly, we give two classical theorems, they are useful to proof theorems in this paper.

Lemma 2.1 (Rouche's theorem) For any two complex-valued functions $f(x)$ and $g(x)$,

1) If they are holomorphic inside some region $D$ with closed contour $C$

2) If $|f(x)|>|g(x)|$ holds on $C$

Then $f(x)$ and $f(x)+g(x)$ have the same number of zeros inside $C$, where each zero is counted as many times as its multiplicity.

Lemma 2.2 (Taylor theorem) If the function $f(x)$ exists the $n t h$ order continuous derivative on $[a, b]$, and the function $f(x)$ exists the $(n+1)$ th order derivative on $(a, b)$, then for any given $x, x_{0} \in[a, b]$, at least one point $\xi \in(a, b)$ exists, such that

$$
\begin{aligned}
f(x)= & f\left(x_{0}\right)+f^{\prime}\left(x_{0}\right)\left(x-x_{0}\right)+\frac{f^{\prime \prime}\left(x_{0}\right)}{2 !}\left(x-x_{0}\right)^{2}+\cdots \\
& +\frac{f^{(n)}\left(x_{0}\right)}{n !}\left(x-x_{0}\right)^{n}+\frac{f^{(n+1)}(\xi)}{(n+1) !}\left(x-x_{0}\right)^{n+1}
\end{aligned}
$$

Then, let $\phi$ and $\psi$ be the solutions of (1), and they satisfy the following conditions:

$$
\phi(0, \lambda)=1, \quad \phi^{\prime}(0, \lambda)=0, \psi(0, \lambda)=0, \psi^{\prime}(0, \lambda)=1
$$

By [5], we have

$$
\begin{gathered}
\phi(x, \lambda)=\cos (\rho x)+\frac{\sin (\rho x)}{2 \rho} \int_{0}^{x} q(t) \mathrm{d} t+\frac{\cos (\rho x)}{\rho^{2}} q_{1}(x)+o\left(\frac{\mathrm{e}^{|\tau| x}}{|\rho|^{3}}\right) \\
\psi(x, \lambda)=\frac{\sin (\rho x)}{\rho}-\frac{\cos (\rho x)}{2 \rho^{2}} \int_{0}^{x} q(t) \mathrm{d} t+o\left(\frac{\mathrm{e}^{|\tau| x}}{|\rho|^{3}}\right)
\end{gathered}
$$

where $\rho=\sqrt{\lambda}=\sigma+i \tau, \sigma, \tau \in R$ and

$$
\begin{gathered}
q_{1}(x)=\frac{q(x)-q(0)}{4}-\frac{1}{8}\left(\int_{0}^{x} q(t) \mathrm{d} t\right)^{2} \\
Q(x)=\int_{0}^{x} q(t) \mathrm{d} t
\end{gathered}
$$

Let a nontrivial solution of (1) be

$$
y(x, \lambda)=c_{1}(\lambda) \phi(x, \lambda)+c_{2}(\lambda) \psi(x, \lambda)
$$

In the following, we will discuss the problem (1), (2), (5); (1), (3), (4) and (1), (3), (5) separately.

\section{Uniqueness and Reconstruction Problem of (1), (2), (5)}

By (2), (5) and (11), we have 


$$
\begin{gathered}
c_{1}(\lambda)\left[\phi(0, \lambda)-\int_{0}^{\pi} \phi(x, \lambda) \mu_{0}(x) \mathrm{d} x\right]+c_{2}(\lambda)\left[\psi(0, \lambda)-\int_{0}^{\pi} \psi(x, \lambda) \mu_{0}(x) \mathrm{d} x\right]=0 \\
c_{1}(\lambda)\left[\phi^{\prime}(\pi, \lambda)-\int_{0}^{\pi} \phi(x, \lambda) v_{\pi}(x) \mathrm{d} x\right]+c_{2}(\lambda)\left[\psi^{\prime}(\pi, \lambda)-\int_{0}^{\pi} \psi(x, \lambda) v_{\pi}(x) \mathrm{d} x\right]=0
\end{gathered}
$$

So the eigenfunction of (1), (2), (5) is

$$
\Delta(\lambda)=\left|\begin{array}{ll}
\phi(0, \lambda)-\int_{0}^{\pi} \phi(x, \lambda) \mu_{0}(x) \mathrm{d} x & \psi(0, \lambda)-\int_{0}^{\pi} \psi(x, \lambda) \mu_{0}(x) \mathrm{d} x \\
\phi^{\prime}(\pi, \lambda)-\int_{0}^{\pi} \phi(x, \lambda) v_{\pi}(x) \mathrm{d} x & \psi^{\prime}(\pi, \lambda)-\int_{0}^{\pi} \psi(x, \lambda) v_{\pi}(x) \mathrm{d} x
\end{array}\right|
$$

Without losing generality, let's suppose

$$
\begin{gathered}
c_{1}(\lambda)=-\psi(0, \lambda)+\int_{0}^{\pi} \psi(x, \lambda) \mu_{0}(x) \mathrm{d} x \\
c_{2}(\lambda)=\phi(0, \lambda)-\int_{0}^{\pi} \phi(x, \lambda) \mu_{0}(x) \mathrm{d} x
\end{gathered}
$$

Let $\lambda_{n}$ is the eigenvalue of the problem, just then

$$
y\left(x, \lambda_{n}\right)=c_{1}\left(\lambda_{n}\right) \phi\left(x, \lambda_{n}\right)+c_{2}\left(\lambda_{n}\right) \psi\left(x, \lambda_{n}\right)
$$

is the corresponding characteristic function. Let $x_{n}^{j}$ be nodes of the characteristic function.

Lemma 3.1 As $n$ is large enough, the property of eigenvalues $\lambda_{n}$ for (1), (2), (5) is listed:

$$
\rho_{n}=\frac{2 n+1}{2}, n \in Z
$$

Proof. Using (7), (8) and (14), as $|\rho| \rightarrow \infty$, we have

$$
\begin{aligned}
\Delta(\lambda)= & \frac{\sin (\rho \pi)}{\rho}\left[\frac{1}{2} Q(\pi)-\mu_{0}(0)\right]+\frac{\cos (\rho \pi)}{\rho^{2}}\left[\mu_{0}^{\prime}(0)-\frac{1}{2} q(\pi)+v_{\pi}(\pi)\right. \\
& \left.+\frac{1}{2} Q(\pi) \mu_{0}(0)\right]+\frac{\cos ^{2}(\rho \pi)}{\rho^{2}}\left[\frac{1}{2} Q(\pi) \mu_{0}(\pi)-\mu_{0}^{\prime}(\pi)\right] \\
& -\frac{1}{\rho^{2}}\left[\frac{1}{2} \mu_{0}(\pi) Q(\pi)+v_{\pi}(0)\right]+\cos (\rho \pi)+o\left(\frac{\mathrm{e}^{2|\tau| \pi}}{|\rho|^{3}}\right)
\end{aligned}
$$

If $\lambda=\rho^{2}$ is eigenvalue of Sturm-Liouville operator (1), (2), (5), as $|\rho| \rightarrow \infty$, we have

$$
\cos (\rho \pi)=0
$$

so we obtain (18).

Lemma 3.2 As $n$ is large enough, the characteristic function $y\left(x, \lambda_{n}\right)$ of (1), (2), (5) has $n-1$ nodal points on $[0, \pi]$ :

$$
x_{n}^{j}=\frac{2 j \pi}{2 n+1}-\frac{4 \mu_{0}(0)}{(2 n+1)^{2}}+\frac{2}{(2 n+1)^{2}} Q\left(x_{n}^{j}\right)+o\left(\frac{1}{n^{3}}\right)
$$

Proof. From (7) and (8), we can get the following formula:

$$
y\left(x, \lambda_{n}\right)=\frac{\sin \left(\rho_{n} x\right)}{\rho_{n}}+\frac{\cos \left(\rho_{n} x\right)}{\rho_{n}^{2}}\left[\mu_{0}(0)-\frac{1}{2} Q(x)\right]+o\left(\frac{1}{\left|\rho_{n}\right|^{3}}\right)
$$


for large enough $n, \quad n \geq N_{0}$, we obtain that $n t h$ characteristic function $y\left(x, \lambda_{n}\right)$ has $n-1$ zeros $x_{n}^{j}$ on $[0, \pi],(j=1,2, \cdots, n-1)$. By $y\left(x_{n}^{j}, \lambda_{n}\right)=0$ we have

$$
\tan \left(\rho_{n} x_{n}^{j}\right)=\frac{Q\left(x_{n}^{j}\right)}{2 \rho_{n}}-\frac{\mu_{0}(0)}{\rho_{n}}+o\left(\frac{1}{\left|\rho_{n}\right|^{2}}\right)
$$

By Lemma 2.2, as $n \rightarrow \infty$, we get

$$
\rho_{n} x_{n}^{j}=j \pi+\frac{Q\left(x_{n}^{j}\right)}{2 \rho_{n}}-\frac{\mu_{0}(0)}{\rho_{n}}+o\left(\frac{1}{\left|\rho_{n}\right|^{2}}\right)
$$

which implies

$$
x_{n}^{j}=\frac{j \pi}{\rho_{n}}+\frac{Q\left(x_{n}^{j}\right)}{2 \rho_{n}^{2}}-\frac{\mu_{0}(0)}{\rho_{n}^{2}}+o\left(\frac{1}{\left|\rho_{n}\right|^{3}}\right)
$$

Theorem 3.3 For $x \in[0, \pi]$, let $\left\{x_{n}^{j}\right\} \subset X$, and $\lim _{n \rightarrow \infty} x_{n}^{j}=x$. Then the following finite limit exists and the corresponding equality holds:

$$
\lim _{n \rightarrow \infty}\left[\left(\frac{2 n+1}{2}\right)^{2} x_{n}^{j}-\frac{2 n+1}{2} j \pi\right]:=f(x)
$$

and

$$
f(x)=\frac{1}{2} Q(x)-\mu_{0}(0)
$$

Proof. By (21) and $\lim _{n \rightarrow \infty} x_{n}^{j}=x$. For any integer $n$, we have

$$
\begin{aligned}
& \lim _{n \rightarrow \infty}\left[\left(\frac{2 n+1}{2}\right)^{2} x_{n}^{j}-\frac{2 n+1}{2} j \pi\right] \\
& =\lim _{n \rightarrow \infty}\left[\frac{1}{2} Q\left(x_{n}^{j}\right)-\mu_{0}(0)\right]=\frac{1}{2} Q(x)-\mu_{0}(0):=f(x)
\end{aligned}
$$

Theorem 3.4 Let $X_{0} \subset X$ be a dense subset in $(0, \pi)$, then $X_{0}$ can uniquely determine the potential function $q(x)$. And we have the algorithm below:

1) For a subset $X_{0}$, and for each $x \in[0, \pi],\left\{x_{n}^{j}\right\} \subset X_{0}$, then $\lim _{n \rightarrow \infty} x_{n}^{j}=x$.

2) By (28) we get

$$
q(x)=2 f^{\prime}(x)
$$

Proof. For a given $X_{0}$, The conclusion is obvious.

Next, let us continue to consider the inverse nodal problems for (1), (3), (4).

\section{Uniqueness and Reconstruction Problem of (1), (3), (4)}

In similar approach to Part 3, we get the eigenfunction of (1), (3), (4) is

$$
\Delta(\lambda)=\left|\begin{array}{ll}
\phi^{\prime}(0, \lambda)-\int_{0}^{\pi} \phi(x, \lambda) v_{0}(x) \mathrm{d} x & \psi^{\prime}(0, \lambda)-\int_{0}^{\pi} \psi(x, \lambda) v_{0}(x) \mathrm{d} x \\
\phi(\pi, \lambda)-\int_{0}^{\pi} \phi(x, \lambda) \mu_{\pi}(x) \mathrm{d} x & \psi(\pi, \lambda)-\int_{0}^{\pi} \psi(x, \lambda) \mu_{\pi}(x) \mathrm{d} x
\end{array}\right|
$$


Now, let's suppose

$$
\begin{gathered}
c_{1}(\lambda)=-\frac{1}{\rho} \psi^{\prime}(0, \lambda)+\frac{1}{\rho} \int_{0}^{\pi} \psi(x, \lambda) v_{0}(x) \mathrm{d} x \\
c_{2}(\lambda)=\frac{1}{\rho} \phi^{\prime}(0, \lambda)-\frac{1}{\rho} \int_{0}^{\pi} \phi(x, \lambda) v_{0}(x) \mathrm{d} x
\end{gathered}
$$

Using (7), (8) and (30), we have

$$
\begin{aligned}
\Delta(\lambda)= & \frac{\cos (\rho \pi)}{\rho^{2}}\left[\mu_{\pi}^{\prime}(\pi)+v_{0}(0)-q_{1}(\pi)-\frac{1}{2} Q(\pi) \mu_{\pi}(\pi)\right] \\
& +\frac{\sin (\rho \pi)}{\rho}\left[\mu_{\pi}(\pi)-\frac{1}{2} Q(\pi)\right]-\frac{1}{\rho^{2}}\left[\mu_{\pi}^{\prime}(0)+v_{0}(\pi)\right] \\
& -\cos (\rho \pi)+o\left(\frac{\mathrm{e}^{2|\tau| \pi}}{|\rho|^{3}}\right)
\end{aligned}
$$

For the characteristic function (17), as $n \rightarrow \infty$, we get

$$
y\left(x, \lambda_{n}\right)=-\frac{\cos \left(\rho_{n} x\right)}{\rho_{n}}-\frac{\sin \left(\rho_{n} x\right)}{2 \rho_{n}^{2}} Q(x)+o\left(\frac{1}{\left|\rho_{n}\right|^{3}}\right)
$$

Then, we can get some conclusions about the problem (1), (3), (4), we will list them without proof.

Lemma 4.1 As $n$ is large enough, the property of eigenvalues $\lambda_{n}$ for (1), (3), (4) is the same as (18).

Lemma 4.2 As $n$ is large enough, the characteristic function $y\left(x, \lambda_{n}\right)$ of (1), (3), (4) has $n-1$ nodal points in $[0, \pi]$ :

$$
x_{n}^{j}=\frac{\pi}{2 n+1}+\frac{2 j \pi}{2 n+1}-\frac{2}{Q(x)}+o\left(\frac{1}{n^{3}}\right)
$$

Theorem 4.3 For $x \in[0, \pi]$, let $\left\{x_{n}^{j}\right\} \subset X$ and $\lim _{n \rightarrow \infty} x_{n}^{j}=x$. Then the following finite limit exists and the corresponding equality holds:

$$
\lim _{n \rightarrow \infty}\left[(2 n+1) Q(x) x_{n}^{j}-2 j \pi Q(x)+2(2 n+1)\right]:=f(x)
$$

and

$$
f(x)=\pi Q(x)
$$

Theorem 4.4 Let $X_{0} \subset X$ be a dense subset in $(0, \pi)$, then $X_{0}$ can uniquely determine the potential function $q(x)$. And we have the algorithm below:

1) For a subset $X_{0}$, and for each $x \in[0, \pi],\left\{x_{n}^{j}\right\} \subset X_{0}$, then $\lim _{n \rightarrow \infty} x_{n}^{j}=x$.

2) By (37) we get

$$
q(x)=\frac{1}{\pi} f^{\prime}(x)
$$

\section{Uniqueness and Reconstruction Problem of (1), (3), (5)}

Similarly, the eigenfunction of (1), (3), (5) is 


$$
\Delta(\lambda)=\left|\begin{array}{ll}
\phi^{\prime}(0, \lambda)-\int_{0}^{\pi} \phi(x, \lambda) v_{0}(x) \mathrm{d} x & \psi^{\prime}(0, \lambda)-\int_{0}^{\pi} \psi(x, \lambda) v_{0}(x) \mathrm{d} x \\
\phi^{\prime}(\pi, \lambda)-\int_{0}^{\pi} \phi(x, \lambda) v_{\pi}(x) \mathrm{d} x & \psi^{\prime}(\pi, \lambda)-\int_{0}^{\pi} \psi(x, \lambda) v_{\pi}(x) \mathrm{d} x
\end{array}\right|
$$

Without losing generality, we still assume (31) and (32) hold in this case.

Lemma 5.1 As $n$ is large enough, the property of eigenvalues $\lambda_{n}$ for (1), (3), (5) is listed:

$$
\begin{aligned}
& \rho_{n}=n+\frac{1}{n \pi} \frac{(-1)^{n}\left[\frac{1}{2} v_{0}(\pi) Q(\pi)+v_{\pi}^{\prime}(0)\right]-(-1)^{n} \cos ^{2}\left(\varepsilon_{n} \pi\right)\left[\frac{1}{2} v_{0}(\pi) Q(\pi)-v_{0}^{\prime}(\pi)\right]}{q_{1}(\pi)-\frac{1}{2} q(\pi)-v_{0}(0)+v_{\pi}(\pi)} \\
& -\frac{1}{n \pi} \frac{\cos \left(\varepsilon_{n} \pi\right)\left[\frac{1}{2} v_{0}(0) Q(\pi)+v_{\pi}^{\prime}(\pi)-\frac{1}{2} v_{\pi}(\pi) Q(\pi)-q_{1}^{\prime}(\pi)+v_{0}^{\prime}(0)\right]}{q_{1}(\pi)-\frac{1}{2} q(\pi)-v_{0}(0)+v_{\pi}(\pi)} \\
& +o\left(\frac{1}{n^{2}}\right), n \in Z \\
& \Delta(\lambda)=\frac{\sin (\rho \pi)}{\rho}\left[q_{1}(\pi)-\frac{1}{2} q(\pi)-v_{0}(0)+v_{\pi}(\pi)\right] \\
& +\frac{\cos (\rho \pi)}{\rho^{2}}\left[\frac{1}{2} Q(\pi) v_{0}(0)+v_{\pi}^{\prime}(\pi)-\frac{1}{2} Q(\pi) v_{\pi}(\pi)-q_{1}^{\prime}(\pi)+v_{0}^{\prime}(0)\right] \\
& +\frac{\cos ^{2}(\rho \pi)}{\rho^{2}}\left[\frac{1}{2} v_{0}(\pi) Q(\pi)-v_{0}^{\prime}(\pi)\right] \\
& -\frac{1}{\rho^{2}}\left[\frac{1}{2} v_{0}(\pi) Q(\pi)+v_{\pi}^{\prime}(0)\right]+o\left(\frac{\mathrm{e}^{2|\tau| \pi}}{|\rho|^{3}}\right)
\end{aligned}
$$

Let

$$
\rho^{2} \Delta(\lambda)=h_{1}(\lambda)+h_{2}(\lambda)
$$

where

$$
\begin{gathered}
h_{1}(\lambda)=\rho \sin (\rho \pi)\left[q_{1}(\pi)-\frac{1}{2} q(\pi)-v_{0}(0)+v_{\pi}(\pi)\right] \\
h_{2}(\lambda)=\cos (\rho \pi)\left[\frac{1}{2} v_{0}(0) Q(\pi)+v_{\pi}^{\prime}(\pi)-\frac{1}{2} v_{\pi}(\pi) Q(\pi)-q_{1}^{\prime}(\pi)+v_{0}^{\prime}(0)\right] \\
+\cos ^{2}(\rho \pi)\left[\frac{1}{2} Q(\pi) v_{0}(\pi)-v_{0}^{\prime}(\pi)\right] \\
-\left[\frac{1}{2} Q(\pi) v_{0}(\pi)+v_{\pi}^{\prime}(0)\right]+o\left(\frac{\mathrm{e}^{2|\tau| \pi}}{|\rho|}\right)
\end{gathered}
$$

In order to accurately estimate eigenvalues $\lambda_{n}$, for given region $r_{n}(\delta)=\{\rho:|\rho-n|=\delta\}$, as radius $\delta$ is small enough and $\delta>0$, we get

$$
\left|h_{1}(\lambda)\right|>\left|h_{2}(\lambda)\right|
$$

So by Lemma 2.1, as $n$ is large enough, we have that $\Delta(\lambda)$ has one zero in- 
side $r_{n}(\delta)$, denote it $\rho_{n} . \delta>0$ is arbitrary, so

$$
\rho_{n}=n+\varepsilon_{n}, \quad \varepsilon_{n}=o(1) \text { as } n \rightarrow \infty
$$

Substituting (46) into (41), we get

$$
\begin{aligned}
0= & \Delta\left(\lambda_{n}\right)=\left(n+\varepsilon_{n}\right) \sin \left[\left(n+\varepsilon_{n}\right) \pi\right]\left[q_{1}(\pi)-\frac{1}{2} q(\pi)-v_{0}(0)+v_{\pi}(\pi)\right] \\
+ & \cos \left[\left(n+\varepsilon_{n}\right) \pi\right]\left[\frac{1}{2} Q(\pi) v_{0}(0)+v_{\pi}^{\prime}(\pi)-\frac{1}{2} Q(\pi) v_{\pi}(\pi)-q_{1}^{\prime}(\pi)+v_{0}^{\prime}(0)\right] \\
+ & \cos ^{2}\left[\left(n+\varepsilon_{n}\right) \pi\right]\left[\frac{1}{2} Q(\pi) v_{0}(\pi)-v_{0}^{\prime}(\pi)\right] \\
- & {\left[\frac{1}{2} Q(\pi) v_{0}(\pi)+v_{\pi}^{\prime}(0)\right]+o\left(\frac{1}{n}\right) } \\
& 0=n \sin \left(\varepsilon_{n} \pi\right)\left[q_{1}(\pi)-\frac{1}{2} q(\pi)-v_{0}(0)+v_{\pi}(\pi)\right] \\
& +\cos \left(\varepsilon_{n} \pi\right)\left[\frac{1}{2} v_{0}(0) Q(\pi)+v_{\pi}^{\prime}(\pi)-\frac{1}{2} v_{\pi}(\pi) Q(\pi)-q_{1}^{\prime}(\pi)+v_{0}^{\prime}(0)\right] \\
& +(-1)^{n} \cos ^{2}\left(\varepsilon_{n} \pi\right)\left[\frac{1}{2} v_{0}(\pi) Q(\pi)-v_{0}^{\prime}(\pi)\right] \\
& \quad(-1)^{n}\left[\frac{1}{2} v_{0}(\pi) Q(\pi)+v_{\pi}^{\prime}(0)\right]+o(1)
\end{aligned}
$$

Using (48), we obtain

$$
\begin{aligned}
\varepsilon_{n}= & \frac{1}{n \pi} \frac{(-1)^{n}\left[\frac{1}{2} v_{0}(\pi) Q(\pi)+v_{\pi}^{\prime}(0)\right]-(-1)^{n} \cos ^{2}\left(\varepsilon_{n} \pi\right)\left[\frac{1}{2} v_{0}(\pi) Q(\pi)-v_{0}^{\prime}(\pi)\right]}{q_{1}(\pi)-\frac{1}{2} q(\pi)-v_{0}(0)+v_{\pi}(\pi)} \\
& -\frac{1}{n \pi} \frac{\cos \left(\varepsilon_{n} \pi\right)\left[\frac{1}{2} v_{0}(0) Q(\pi)+v_{\pi}^{\prime}(\pi)-\frac{1}{2} v_{\pi}(\pi) Q(\pi)-q_{1}^{\prime}(\pi)+v_{0}^{\prime}(0)\right]}{q_{1}(\pi)-\frac{1}{2} q(\pi)-v_{0}(0)+v_{\pi}(\pi)} \\
& +o\left(\frac{1}{n^{2}}\right), n \in Z
\end{aligned}
$$

It can be seen from (46) and (49), the Lemma holds.

Lemma 5.2 As $n$ is large enough, the characteristic function $y\left(x, \lambda_{n}\right)$ of (1), (3), (5) has $n-1$ nodal points in $[0, \pi]$ :

$$
\begin{gathered}
x_{n}^{j}=\frac{\pi}{2 n}+\frac{j \pi}{n}-\frac{2}{Q(x)} \\
-\frac{j}{n^{3}}\left\{\frac{(-1)^{n}\left[\frac{1}{2} v_{0}(\pi) Q(\pi)+v_{\pi}^{\prime}(0)\right]-(-1)^{n} \cos ^{2}\left(\varepsilon_{n} \pi\right)\left[\frac{1}{2} v_{0}(\pi) Q(\pi)-v_{0}^{\prime}(\pi)\right]}{q_{1}(\pi)-\frac{1}{2} q(\pi)-v_{0}(0)+v_{\pi}(\pi)}\right\} \\
+\frac{j}{n^{3}}\left\{\frac{\cos \left(\varepsilon_{n} \pi\right)\left[\frac{1}{2} v_{0}(0) Q(\pi)+v_{\pi}^{\prime}(\pi)-\frac{1}{2} v_{\pi}(\pi) Q(\pi)-q_{1}^{\prime}(\pi)+v_{0}^{\prime}(0)\right]}{q_{1}(\pi)-\frac{1}{2} q(\pi)-v_{0}(0)+v_{\pi}(\pi)}\right\} \\
+o\left(\frac{1}{n^{3}}\right), n \in Z \quad
\end{gathered}
$$


Proof. From (7) and (8), as $n \rightarrow \infty$, we have

$$
y\left(x, \lambda_{n}\right)=-\frac{\cos \left(\rho_{n} x\right)}{\rho_{n}}-\frac{\sin \left(\rho_{n} x\right)}{2 \rho_{n}^{2}} Q(x)+o\left(\frac{1}{\left|\rho_{n}\right|^{3}}\right)
$$

Similar to Part 3 and Part 4, as $n$ is large enough and $n \geq N_{0}$, we have that $n$th characteristic function $y\left(x, \lambda_{n}\right)$ has $n-1$ zeros $x_{n}^{j}$ in $[0, \pi]$. From $y\left(x_{n}^{j}, \lambda_{n}\right)=0$, we have

$$
\tan \left(\rho_{n} x_{n}^{j}\right)=-\frac{2 \rho_{n}}{Q\left(x_{n}^{j}\right)}+o\left(\frac{1}{\left|\rho_{n}\right|^{2}}\right)
$$

Applying Lemma 2.2, we have

$$
x_{n}^{j}=\frac{\pi}{2 \rho_{n}}+\frac{j \pi}{\rho_{n}}-\frac{2}{Q\left(x_{n}^{j}\right)}+o\left(\frac{1}{\left|\rho_{n}\right|^{3}}\right)
$$

From (40), we can get

$$
\rho_{n}^{-1}=\frac{1}{n}-\frac{C_{\mu}}{n^{3}}+o\left(\frac{1}{n^{4}}\right), \quad \rho_{n}^{-2}=\frac{1}{n^{2}}-\frac{2 C_{\mu}}{n^{4}}+o\left(\frac{1}{n^{5}}\right)
$$

where

$$
\begin{gathered}
C_{\mu}=\frac{1}{\pi}\left\{\frac{(-1)^{n}\left[\frac{1}{2} v_{0}(\pi) Q(\pi)+v_{\pi}^{\prime}(0)\right]-(-1)^{n} \cos ^{2}\left(\varepsilon_{n} \pi\right)\left[\frac{1}{2} v_{0}(\pi) Q(\pi)-v_{0}^{\prime}(\pi)\right]}{q_{1}(\pi)-\frac{1}{2} q(\pi)-v_{0}(0)+v_{\pi}(\pi)}\right\} \\
-\frac{1}{\pi}\left\{\frac{\cos \left(\varepsilon_{n} \pi\right)\left[\frac{1}{2} v_{0}(0) Q(\pi)+v_{\pi}^{\prime}(\pi)-\frac{1}{2} v_{\pi}(\pi) Q(\pi)-q_{1}^{\prime}(\pi)+v_{0}^{\prime}(0)\right]}{q_{1}(\pi)-\frac{1}{2} q(\pi)-v_{0}(0)+v_{\pi}(\pi)}\right\}
\end{gathered}
$$

Thus we can obtain (50).

Theorem 5.3 For $x \in[0, \pi]$, let $\left\{x_{n}^{j}\right\} \subset X$, and $\lim _{n \rightarrow \infty} x_{n}^{j}=x$. Then the following finite limit exists and the corresponding equality holds:

$$
\lim _{n \rightarrow \infty}\left[n^{2} Q(x) x_{n}^{j}-\frac{1}{2} j \pi n Q(x)-n j \pi Q(x)+2 n^{2}\right]:=f(x)
$$

and

$$
\begin{aligned}
f(x)= & \frac{x}{\pi} Q(x)\left\{\frac{\cos \left(\varepsilon_{n} \pi\right)\left[\frac{1}{2} v_{0}(0) Q(\pi)+v_{\pi}^{\prime}(\pi)-\frac{1}{2} v_{\pi}(\pi) Q(\pi)-q_{1}^{\prime}(\pi)+v_{0}^{\prime}(0)\right]}{q_{1}(\pi)-\frac{1}{2} q(\pi)-v_{0}(0)+v_{\pi}(\pi)}\right\} \\
& -\frac{x}{\pi} Q(x)\left\{-\frac{(-1)^{n}\left[\frac{1}{2} v_{0}(\pi) Q(\pi)+v_{\pi}^{\prime}(0)\right]-(-1)^{n} \cos ^{2}\left(\varepsilon_{n} \pi\right)\left[\frac{1}{2} v_{0}(\pi) Q(\pi)-v_{0}^{\prime}(\pi)\right]}{q_{1}(\pi)-\frac{1}{2} q(\pi)-v_{0}(0)+v_{\pi}(\pi)}\right\}
\end{aligned}
$$

Proof. Using (50) and $\lim _{n \rightarrow \infty} x_{n}^{j}=x$, we have 


$$
\begin{aligned}
& \lim _{n \rightarrow \infty}\left[n^{2} Q(x) x_{n}^{j}-\frac{1}{2} j \pi n Q(x)-n j \pi Q(x)+2 n^{2}\right] \\
& =\lim _{n \rightarrow \infty}\left\{\frac{x}{\pi} Q\left(x_{n}^{j}\right) \frac{\cos \left(\varepsilon_{n} \pi\right)\left[\frac{1}{2} v_{0}(0) Q(\pi)+v_{\pi}^{\prime}(\pi)-\frac{1}{2} v_{\pi}(\pi) Q(\pi)-q_{1}^{\prime}(\pi)+v_{0}^{\prime}(0)\right]}{q_{1}(\pi)-\frac{1}{2} q(\pi)-v_{0}(0)+v_{\pi}(\pi)}\right. \\
& \left.-\frac{x}{\pi} Q\left(x_{n}^{j}\right) \frac{(-1)^{n}\left[\frac{1}{2} v_{0}(\pi) Q(\pi)+v_{\pi}^{\prime}(0)\right]-(-1)^{n} \cos ^{2}\left(\varepsilon_{n} \pi\right)\left[\frac{1}{2} v_{0}(\pi) Q(\pi)-v_{0}^{\prime}(\pi)\right]}{q_{1}(\pi)-\frac{1}{2} q(\pi)-v_{0}(0)+v_{\pi}(\pi)}\right\} \\
& =\frac{x}{\pi} Q(x)\left\{\frac{\cos \left(\varepsilon_{n} \pi\right)\left[\frac{1}{2} v_{0}(0) Q(\pi)+v_{\pi}^{\prime}(\pi)-\frac{1}{2} v_{\pi}(\pi) Q(\pi)-q_{1}^{\prime}(\pi)+v_{0}^{\prime}(0)\right]}{q_{1}(\pi)-\frac{1}{2} q(\pi)-v_{0}(0)+v_{\pi}(\pi)}\right\} \\
& -\frac{x}{\pi} Q(x)\left\{\frac{(-1)^{n}\left[\frac{1}{2} v_{0}(\pi) Q(\pi)+v_{\pi}^{\prime}(0)\right]-(-1)^{n} \cos ^{2}\left(\varepsilon_{n} \pi\right)\left[\frac{1}{2} v_{0}(\pi) Q(\pi)-v_{0}^{\prime}(\pi)\right]}{q_{1}(\pi)-\frac{1}{2} q(\pi)-v_{0}(0)+v_{\pi}(\pi)}\right\} \\
& =f(x)
\end{aligned}
$$

So we obtain (57).

Theorem 5.4 Let $X_{0} \subset X$ be a dense subset in $(0, \pi)$, then $X_{0}$ can uniquely determine the potential function $q(x)$. And we have the algorithm below:

1) For a subset $X_{0}$, and for each $x \in[0, \pi],\left\{x_{n}^{j}\right\} \subset X_{0}$, then $\lim _{n \rightarrow \infty} x_{n}^{j}=x$.

2) By (57) we get

$$
\frac{2 q(x)+x q^{\prime}(x)}{[q]}=\frac{f^{\prime \prime}(x)}{f(\pi)}
$$

where $[q]=\pi Q(\pi)$.

Proof. For a given $X_{0}$, by (57), we have

$$
\begin{aligned}
f^{\prime \prime}(x)=\left[\frac{2}{\pi} q(x)+\frac{x}{\pi} q^{\prime}(x)\right]\left\{\frac{\cos \left(\varepsilon_{n} \pi\right)\left[\frac{1}{2} v_{0}(0) Q(\pi)+v_{\pi}^{\prime}(\pi)-\frac{1}{2} v_{\pi}(\pi) Q(\pi)-q_{1}^{\prime}(\pi)+v_{0}^{\prime}(0)\right]}{q_{1}(\pi)-\frac{1}{2} q(\pi)-v_{0}(0)+v_{\pi}(\pi)}\right. \\
\left.-\frac{(-1)^{n}\left[\frac{1}{2} v_{0}(\pi) Q(\pi)+v_{\pi}^{\prime}(0)\right]-(-1)^{n} \cos ^{2}\left(\varepsilon_{n} \pi\right)\left[\frac{1}{2} v_{0}(\pi) Q(\pi)-v_{0}^{\prime}(\pi)\right]}{q_{1}(\pi)-\frac{1}{2} q(\pi)-v_{0}(0)+v_{\pi}(\pi)}\right\}
\end{aligned}
$$

So we have (58).

\section{Conclusion}

In this work, we study three inverse nodal problems for the Sturm-Liouville operator with different nonlocal integral conditions. We get the uniqueness theorems. And we present some constructive procedures to solve the inverse nodal 
problems. It makes sense for the theoretical integrity of the inverse nodal problem with nonlocal integral conditions.

\section{Fund}

This work was supported by the National Natural Science Foundation of China (11661059) and the Natural Science Foundation of the Inner Mongolia Autonomous Region of China (2017MS(LH)0103).

\section{Conflicts of Interest}

The authors declare no conflicts of interest regarding the publication of this paper.

\section{References}

[1] Yurko, V.A. (2008) Inverse Nodal Problems for Sturm-Liouville Operators on Star-Type Graphs. Journal of Inverse and Ill-Posed Problems, 16, 715-722. https://doi.org/10.1515/JIIP.2008.044

[2] Levitan, B.M. (1987) Inverse Sturm-Liouville Problems. VNU Science Press, Utrecht. https://doi.org/10.1515/9783110941937

[3] Rundell, W. and Sacks, P. (2004) Numerical Technique for the Inverse Resonance Problem. Journal of Computational and Applied Mathematics, 170, 337-347. https://doi.org/10.1016/j.cam.2004.01.035

[4] Yurko, V.A. (2000) Integral Transforms Connected with Discontinious Boundary Value Problems. Integral Transforms and Special Functions, 10, 141-164. https://doi.org/10.1080/10652460008819282

[5] Yurko, V.A. (2000) Inverse Spectral Problems for Differential Operators and Their Applications. Gordon and Breach, Amsterdam.

[6] Mclaughlin, J.R. (1988) Inverse Spectral Theory Using Nodal Points as Data-A Uniqueness Result. Journal of Differential Equations, 73, 354-362. https://doi.org/10.1016/0022-0396(88)90111-8

[7] Hald, O.H. and Mclaughlin, J.R. (1989) Solutions of Inverse Nodal Problems. Inverse Problems, 5, 307-347.

[8] Yang, X.-F. (2001) A New Inverse Nodal Problem. Journal of Differential Equations, 169, 633-653. https://doi.org/10.1006/jdeq.2000.3911

[9] Yang, X.-F. (1997) A Solutions of the Inverse Nodal Problem. Inverse Problems, 13, 203-213.

[10] Koyunbakan, H. (2008) The Inverse Nodal Problem for a Differential Operator with an Eigenvalue in the Boundary Condition. Applied Mathematics Letters, 21, 1301-1305.

[11] Yang, C.-F. (2013) Inverse Nodal Problems of Discontinuous Sturm-Liouville Operator. Journal of Differential Equations, 254, 1992-2014. https://doi.org/10.1016/j.jde.2012.11.018

[12] Yang, C.-F. (2014) Inverse Nodal Problems for the Sturm-Liouville Operator with a Constant Delay. Journal of Differential Equations, 257, 1288-1306. https://doi.org/10.1016/j.jde.2014.05.011

[13] Yang, C.-F. (2017) Inverse Problems for the Differential Operator on a Graph with Cycles. Journal of Mathematical Analysis and Applications, 445, 1548-1562. 
https://doi.org/10.1016/j.jmaa.2016.01.007

[14] Wang, Y.P. and Yurko, V.A. (2016) On the Inverse Nodal Problems for Discontinuous Sturm-Liouville Operators. Journal of Differential Equations, 260, 4086-4109. https://doi.org/10.1016/j.jde.2015.11.004

[15] Pinasco, J.P. and Scarola, C. (2015) A Nodal Inverse Problem for Second Order Sturm-Liouville Operators with Indefinite Weights. Applied Mathematics and Computation, 256, 819-830. https://doi.org/10.1016/j.amc.2015.01.101

[16] Wei, G.S. and Guo, Y.X. (2018) The Sharp Conditions of the Uniqueness for Inverse Nodal Problems. Journal of Differential Equations, in press.

[17] Hu, Y.-T., Yang, C.-F. and Xu, X.-C. (2017) Inverse Nodal Problems for the Sturm-Liouville Operator with Nonlocal Integral Conditions. Journal of Inverse and Ill-Posed Problems, 25, 799-806. https://doi.org/10.1515/jiip-2017-0017 